\title{
Complex Regional Pain SyndRomes (CRPS) TYPE 1 VALIDATING CASE Histories
}

\begin{abstract}
The treatment of patients with complex regional pain syndrome (CRPS) type 1 is challenging and unpredictable as the condition presents with vascular and neuropathic symptoms after nil or even minor injury to a peripheral nerve. The condition is one of a pain and motor dysfunction. The pathophysiology is not well understood and the relief of symptoms may change from being sympathetically mediated to sympathetically independent during the course of the disease. At any stage physiotherapy has been advocated as the corner stone and most important aspect of treatment in the rehabilitation of these individuals but unfortunately it has been difficult to execute when pain is exacerbated due to allodynia (unbearable to touch or move) and hyperalgesia. Best results have been obtained if the patients are recognised and treated in the early or acute phase and it has been found that through careful assessment and analysis these patients can be recognised by previous events that have occurred in their initial case history. The treatment in the acute stage with physiotherapy modalities such as electrical stimulation and acupuncture will produce an early cessation of the symptoms and prevention of the disease developing into the fully blown CRPS type 1 with irreversible and possibly atrophic consequences. Case histories have been presented that illustrate these important aspects and demonstrate the value of early and the appropriate physiotherapy that may be more successful than other pharmacological and physical interventions in this disease.
\end{abstract}

\section{KEY WORDS: PAIN, DYSFUNCTION, ELECTRICAL STIMULATION, ACUPUNCTURE, REHABILITATION}

\section{INTRODUCTION}

Throughout history often the most profound and sad ordeals of human suffering are experienced during wars. Reflex sympathetic dystrophy (RSD), although unrecognised at the time was first documented during the American Civil War in 1864. The first dent in the armour of complex peripheral pain disorders was made when Mitchell et al (1864) published the book Gunshot Wounds and other injuries to nerves describing the signs and symptoms of peripheral nerve injuries as they were

CORRESPONDENCE TO:

$\mathrm{P}$ Berger

40 East Road

Morningside Manor

Sandton

Tel: (011) 802-1275 (w)

Tel: (011) 802-8072 (h)

Fax: (011) 802-5443

Cell: 082 411-7777 observed in Unionist soldiers at Turners Alne Hospital for Nervous Diseases in Philadelphia.

Complex regional pain syndrome encompasses two disorders. The first is Complex regional pain syndrome type 1 (CRPS 1) according to Mersky and Bogduk (1994) previously known as reflex sympathetic dystrophy (RSD) which refers to a complex disorder that may develop as a consequence of trauma affecting the limbs, without an obvious nerve lesion. "In $10-26 \%$ of patients there may not even be an antecedent event" (Shipton 1993). The second is complex regional pain syndrome 2 according to Glynn (1995) was known as causalgia. In this condition there is a specific injury to a peripheral nerve. The two syndromes are however variations of a similar problem. Both types were renamed with their present nomenclature by the International Association for the Study of Pain (IASP) in 1995 and classified as complex regional pain syndrome (CRPS) types 1 and 2 at the Official Joint Satellite meeting in Budapest in 1999 of the Special Interest Group: Pain and the Sympathetic Nervous System and the Section of the Autonomic Nervous System of the German Neurological Society.

CRPS is one of those conditions that confounded the medical profession since it was first encountered; therefore it may often proceed unrecognised. It was also known by many other names such as: acute atrophy of bone; causalgia; minor causalgia; minor traumatic dystrophy; peripheral acute trophoneurosis, post infarctional sclerodactyly; posttraumatic osteoporosis; post-traumatic spreading neuralgia; post-traumatic sympathalgia; reflex dystrophy of the extremities; reflex neurovascular dystrophy; algoneurodystrophy, Sudeck's atrophy; Sudeck's osteodystrophy; sympathalgia; shoulder-hand syndrome; traumatic angiospasm and traumatic vasospasm (Del Carmen 1991). The 
various names ascribed to this condition indicate that the medical profession is undecided on both its name and the precise cause. These disorders are usually the consequence of an injury that is occasionally minor in nature or "may occur during surgery, fracture or severe hand injury" (Bruehl S et al 2002).

CRPS 1 is unexplained by other peripheral nerve injury and often occurs after unknown aetiology, with a pathophysiological mechanism that is not fully understood, the course of the disease being unpredictable and the treatments producing unproven success with consistent therapies. The new terminology therefore is based on the history, symptoms and clinical findings with no implied pathophysiological mechanism (Scadding 1999).

The clinical constellation of signs and symptoms consists of: severe, unrelenting, shooting, burning, electrical pain in the distribution of the affected nerve with frequent spread to adjoining areas (Devor 1994). The referral is mostly proximal with involvement of the spinal and supraspinal mechanisms with possible spread of the symptoms to the opposite side of the body (mirror pain); decreased range of movement, stiffness, wasting, hypotonia, tremor and fasciculation; sensory changes including hyperaesthesia, hyperalgesia and allodynia; psychological changes such as depression, anxiety, suicidal and unusual behaviour patterns and autonomic changes such as temperature and colour changes, oedema and hyperhidrosis with a possible aversion to touch, heat, cold, wind, vibration and even air conditioning.

The most recent understanding of this condition that demonstrates neuropathic pain defined by Mersky and Bogduk (1994) as pain initiated or caused by a primary lesion/dysfunction in the nervous system) suggests that the lesion/dysfunction may be the initiating cause of the pain. Thus the pain is related to the primary lesion or to a dysfunction of the nervous system (Babbedge et al 1995).

Physiotherapists often encounter CRPS 1 patients, however they may become confused as the normal treatment protocol will not benefit these patients, who will mostly deteriorate with the conventional approach to phy- siotherapy such as passive examination and mobilisation and any treatment that involves pressure on the affected area. It has often been found by physiotherapists that there is indeed a local anatomical area in these conditions that appears to be tender and hyperaesthetic and which demonstrates allodynia and hyperpathia. When these areas do not respond to the normal physiotherapeutic modalities the physiotherapist becomes aware that local treatment alone will not dissipate the problem. A search of other areas must then be carried out in order to explore regions that refer to the painful site - these may involve peripheral and even secondary trigger points (Travell and Simons 1983). The pain referral from these areas appears to be nondermatomal in nature and to emanate from local tissue and nerves. It may be diffuse and involve the whole limb and usually refers proximally. It appears that there may be aberrations in the neurophysiology, neuropharmacolgy and neuroanatomy of these regions and it is noted that this is almost the norm after nerve damage in animal models of nociception (Babbedge et al 1995).

Under the new classification of CRPS according to Roberts (1986) it is designated as pain that is responsive or non-responsive to sympathetic block (i.e. sympathetically maintained pain or sympathetically independent pain). This can also occur in an individual whether or not the complaint is a CRPS disorder. In positive responders the degree of pain relief may be quite variable, some demonstrating marked improvement while others show little benefit. The degree of responsiveness can also change over time in any one patient (Torebjork et al 1995).

These patients have chronic pain and the pain in CRPS, repetitive strain injuries (RSI) and inflamed joints usually develops from tissue damage and from various types of nerve disturbances and therefore understanding the symptoms and relief obtained from inflammatory joint disease and RSI may strengthen our knowledge and treatment of CRPS. Kidd et al (1996) refers to pain sensations arising from inflamed tissues and joints that are mediated in the first instance by primary sensory (afferent) fibres that link peripheral receptors with second-order neurons in the spinal cord. These afferent fibres are inherently dynamic structures having an ability to vary responses to a broad range of stimuli including noxious stimuli (Rang and Bevan 1994). These sensory fibres become sensitised in the presence of inflammation and show increased responsiveness to stimuli that are applied to the tissue involved.

Whether one evaluates CRPS patients as being in stages or grades it is apparent that this condition is one of PAIN creating a MOTOR dysfunction that will inevitably become irreversible if left untreated or unrecognised in its early stages. This may cause permanent disability in the limb with general disablement in the life situation but if treated as soon as possible, will then respond rapidly to treatment.

The earliest manifestation of CRPS 1 may present immediately after surgery or POP, usually with sharp, burning, throbbing, twinging, spreading and/or relentless pain and can develop within hours, days or weeks after the original insult to the nerve. Unfortunately the condition is rarely diagnosed and often mistreated.

In a case history of a female, aged 50 years, after fracture of the distal radius four months previously, the patient mentioned that she had immediate pain since the plaster of paris (POP) and "could not permit the limb to hang down due to the pain" and therefore kept the elbow and shoulder splinted. She had many months of conventional physiotherapy in which she was forced to move through the pain. This contributed to spread of pain to the elbow, shoulder, anterior chest wall and cervical musculature and a full-blown manifestation of the dystrophic stage of CRPS 1 occurred. If the pain had been blocked immediately and perhaps the cast reset she would have been able to move, circulation would have been restored and the condition would have been alleviated and or prevented.

Another case history presented is of a sportsman, aged 28 years who had arthroscopic surgery (lateral menisectomy) on the left knee three weeks previously and then had two further 
arthroscopies during this period to investigate continuing oedema and lack of mobility. The patient was referred for mobilisation and conventional rehabilitation which then aggravated his condition. On examination the patient did not complain of pain but rather severe stiffness, the patella appeared captured, bound down in adhesive tissue and oedematous, secondary muscle spasm occurred in the hamstring and calf and severe wasting of the adductors and quadriceps was evident. The history commenced six months prior to the initial arthroscopy when the patient had a traumatic incident on the sports field and the knee became increasingly uncomfortable and then also a preceding history of arthroscopy on the right knee seven years previously. It would have been pertinent to review the history of previous surgery to the opposite leg and possibly inadequate relief of symptoms from the original injury six months previously. It is also suggested that if the added arthroscopies were avoided and a holistic approach of gentle rehabilitation, electrical stimulation and acupuncture were applied this patient may have made an uneventful recovery.

It is important to evaluate the history, symptoms and clinical findings in order to obtain an accurate diagnosis and avoid incorrect or mistreatment of these patients.

On examination of the process that occurs in CRPS 1 it is accepted that there is probably some contusion or compression of neural tissue leading to neuropathic inflammation and long-term potentiation and sensitisation. In electrogenesis, chemicals surrounding peripheral nociceptors sensitise them to thermal and mechanical stimuli and the inflammation in or around the nerve cell/ganglion introduces new chemical messengers that alter the neural response as is seen in ectopia (ectopic discharge from a neuroma). The influence of the sympathetic nervous system depends on the linkage between peripheral nociceptive signal generation and efferent responses from spinal neurones (Birklein et al 1994). It has been observed that a chronic peripheral nociceptive stimulus leads to widespread central spinal sensitisation and efferent discharge. Loss of input to the dorsal horn may precipitate deafferentation hypersensitivity that may manifest as "burning" pain among others. Patients with spinal cord injury often manifest with this phenomenon (Bennett 1994).

In the latest research findings on the aetiology, "the normal spontaneous discharge of dorsal root ganglion neurons is greatly enhanced by peripheral nerve injury and may be further increased by sympathetic stimulation" albeit transiently (Kirk 1974; Devor et al 1994).

Stanton Hicks, at the World Institute of Pain Congress in Istanbul (2001) argued that sympathetically maintained pain (SMP) is not due to a hyper/ hypoactive sympathetic nervous system but due to circulating catecholamines in decreased or normal levels (Stanton Hicks 2001). SMP may also present with no history and even no pain symptoms (? stiffness). The emotions that are prevalent in these patients are anxiety, fear, depression and anger (see case history A). Other factors demonstrated a predisposition in females of $2: 1 ; 10 \%$ widespread repeat presentation; 5\% genetic presentation (dystrophic stage CRPS 1 patient's sister) and 70\% had previous injury with abnormal chronic pain (see case history B). Perpetuating or predisposing factors such as inactivity, protective postures (dystrophic CRPS 1 patient) and splinting may also be present. Other factors involve chemical intolerance, sensitivity to tricyclics and food, irritable bowel syndrome (IBS), fibromyalgia and migraine. The psychological factors that may be influential are fatigue, kinesiophobia, somatic focus and maladaptive limb postures.

In a study by Wasner and Baron (2001) there was a significant correlation between vascular abnormalities and the duration of CRPS 1. The warmer the affected extremity the more acute and the cooler the extremity the more chronic the disease is. Noradrenaline levels were reduced in both the warm and the cold regulation types indicating decreased activity of the sympathetic vasomotor system. Sympathetic dysfunction is expressed by loss or reduced superficial vasomotor control and variable sudomotor function, especially hyperhidrosis early on in the disease.
In another study to analyse impairment of sympathetic vasoconstrictor reflexes it was found that impairment existed exclusively in CRPS patients (24 patients at 5 weeks) as compared with patients post trauma, after external fixation of a distal radius fracture (20 patients at 3.5 days after surgery without signs of CRPS). The study indicated that the additional changes were due to impairment of sympathetic vasocostrictor reflexes and not a simple exaggeration of the post-traumatic inflammation from trauma (Birklein et al 2001).

The initial treatment approach to a patient with pain and lack of mobility is usually conventional physiotherapy however if the patient exhibits any of the above constellation of symptoms and signs of CRPS 1 then the main goal of the treatment is to block the pain cycle, remove or search for the causative factor (inflamed scar, contusion of the big toe) if possible and increase pain-free movement towards full function.

The above cannot occur without patient compliance and this is achieved by explanation and education about the condition and the proposed treatment. The patient is taught that the main aim of treatment is movement of the affected extremity and indeed the whole limb. The physiotherapist will explain that pain will be relieved by electrical stimulation analgesia (ESA) and or acupuncture and that when pain is relieved the mobility will return without fear of increase of pain. If pain should return the patient is encouraged to take control by being given coping strategies such as medication, ESA and or gentle movements in the bath or in a bowl of water to improve circulation and to prevent exacerbation by taking early and effective pain control measures. The patient is encouraged to pace their activities, carefully improving mobility to prevent setbacks.

The patient's preferences are important in deciding the best treatment plan should the patient indicate that touch increases the pain then treatment should avoid irritating the A beta-receptors and should preferably include distal, scalp or micro-system treatment with ear acupuncture, laser or micro-current or other subliminal currents to the 
periphery and transcutaneous electrical nerve stimulation (TENS) either proximal and distal to the affected area or on the spinal segmental or sympathetic region supply to the affected area. Other subliminal currents may also be applied centrally such as Sub-perception Electrical Stimulation (SPES).

The following protocol describes the generalised treatment applied to CRPS 1 patients that has proven over many years to be successful in many patients.

\section{TREATMENT OF THE CRPS PATIENT}

- Scalp acupuncture for relaxation, mobility, unusual pain (burning, shooting)

- Ear acupuncture for the autonomic nervous system, pain relief and mobility

- Local treatment to painful region if possible with electrical stimulation analgesia (ESA) (direct currents, ultrasound)

- Laser for circulation and inflammation

- Constantly encourage active mobility

- Electro-acupuncture superior and inferior to the painful region

- Transcutaneous electrical nerve stimulation (TENS) for home use for at least three days, eight hours per day

- Microcurrent membranes, magnets if appropriate

- Gel, creams, oils if appropriate

- Limb immersed in water with electrical stimulation analgesia (ESA)

- Establish confidence in the treatment

\section{DISCUSSION}

Specific acupuncture points are used on the scalp for relaxation, stress relief and improved mobility and when used with TENS stimulation at a low frequency, will relieve burning (de-afferentation type pain) or unusual pain states. Stress sensitises nociceptors and increases the pain, anxiety and depression that occur as a result of chronic pain states. Other points that are used for pain relief are found in the ear. The ear is a microsystem that represents the whole body including autonomic, pain relief and peripheral regions that will influence the autonomic symptom, increase pain relief and encourage pain free movement in the particular extremity or limb treated.
Electric currents will be applied on the body that may physiologically block the pain (TENS - biphasic, alternating current) or decrease inflammation, relieve pain and improve circulation (direct currents). Direct currents can be used with the limb immersed in water to provide pain relief and improvement in circulation. Micro-currents and laser (subliminal) may also provide relief in hyperaesthesia and improve cell metabolism and newly developed currents such as Sub-perception electrical nerve stimulation (SPES) which is a subliminal current that may be applied centrally (on the ear lobes to target the locus coeruleus) to increase beta endorphins and noradrenaline with effects on the pain and autonomic system.

The following case histories will illustrate early and effective treatment of the CRPS 1 patient.

\section{PATIENT A}

A female of 40 years old was diagnosed with Reflex Sympathetic Dystrophy (RSD) following nerve injury that occurred on $4 / 03 / 02$. She attended her first treatment on 25/03/02.

\section{History and Symptoms}

The symptoms presented were due to an injury three weeks previously in which a garden hose accidentally struck the patient on the side of her lateral lower leg at the level of the peroneal nerve. The patient proffered spontaneously that "I was angry at the time of the incident". The patient was unable to weight bear after the incident; had no active dorsiflexion and the pain extended from the region of the peroneal nerve into the anterior ankle joint. There was slight pain extending into the medial aspect of the thigh. The pain was described as burning, pulling, shooting, hot, there was increased pain at night and the pain visual analogue scale (VAS) score was $7 / 10$. Other remarks made by the patient were "I cannot sleep and my leg is not getting better".

\section{Observation and Examination}

The patient was asked to actively move her foot and the range of motion was noted. There was nil dorsiflexion and all the other movements of the ankle were mimimal, the patient was not comfortable with pressure on the areas of pain and there was no visible injury to be seen in the prescribed area of the pain.

\section{Treatment}

The first treatment consisted of ear and scalp acupuncture. The patient was then asked to move the ankle if possible there was an immediate improvement in dorsiflexion and eversion. A direct current Action Potential Simulation (APS) therapy was applied from the peroneal region into the ankle. TENS was then given for home use to be used for eight hours daily for three consecutive days.

At the second visit the patient recorded a VAS pain score of 3.25/10. The same treatment was repeated as before and electroacupuncture was added by inserting needles into the region near the peroneal nerve and the ankle and attaching a low frequency current to these needles. TENS was continued as a home treatment as the pain was still present.

At the third and last treatment a VAS pain score was recorded of $0 / 10$, dorsiflexion and all other range of movements of the ankle were normal, the patient had discarded her crutches, there was no night pain, the patient was sleeping through the night and no further treatment was necessary.

\section{Discussion}

The patient was referred with the diagnosis of RSD which was the previous nomenclature describing Complex Regional Pain Syndrome type 1. The symptoms were only present for three weeks yet they were becoming increasingly severe.

If treatment were initiated that had increased her pain eg ultrasound, mobilising or even massage - her condition could have exacerbated. However after 2 treatments of TENS a pain blocking modality, distal acupuncture, non-irritating direct electrical current to the local region of pain, the symptoms improved. During the first treatment after the ear acupuncture the patient was able to move the foot into dorsiflexion and eversion without difficulty. After this treatment the patient was also able to ambulate more comfortably. These are indications for the general success of the 
treatment and outcome expectations. After the first treatment there was 50\% improvement in the pain and this was a positive indication that then encouraged use of the local acupuncture in the affected areas without fear of exacerbation. It was also established that this patient was a responder to TENS. According to clinical findings, Berger (1999) maintains that $10 \%$ of patients may be complete responders to TENS without any further intervention necessary. On arrival for the third treatment there was no pain and full movement was obtained and it was decided to cease further treatments. After discussion with this patient she described her mental state at the time of the injury and was able to understand that stress could irritate and exacerbate this injury and then she decided to consult further with a psychologist to deal with various life issues.

\section{PATIENT B}

A female of 11 years with pain in her left lateral ankle joint, the referring diagnosis was CRPS type 1.

\section{History and Symptoms}

The patient presented with generalised pain for the past three weeks in her left lateral ankle joint. During the 2002 holidays in December this patient spent a great deal of the time bare-foot. Pain developed in the ankle and she was referred for physiotherapy that included ultrasound, massage, interferential and exercises for the ankle. Pain increased and was also not alleviated by nonsteroidal anti-inflammatories. She was then referred for pain management. She was sensitive to the touch (hyperaesthetic), non-weight bearing on crutches and could not bear any weight on her foot, she had severe limitation of dorsiflexion and eversion, the pain was burning, sharp, wakes the patient at night, the foot was cold and the pain VAS score varied from 5 to $10 / 10$.

\section{Previous History}

Two years previously the patient had sustained an overuse injury (diagnosed by the doctor) in the ankle and was placed in a plaster of paris cast for four weeks. The pain then settled down until one year later when generalised aches and pains developed in both ankles and orthotics were then prescribed.

\section{Treatment}

The first treatment consisted of ear acupuncture given by laser on the acupoints instead of needle puncture. Laser was used to treat the local ankle region and a direct current (APS therapy) was applied to the whole lower leg to improve the circulation. The patient was also given TENS for home use for three consecutive days for eight hours per day.

At the second treatment the patient reported a varied VAS pain score of 2 $10 / 10$. there was still pain present at night, the foot was still cold and there was a smaller specific region of pain in the lateral collateral ligament region of the ankle. The mother reported a change in the child's personality stating "I have my child back!". The child was less depressed and more cheerful. The treatment was repeated but the direct current was then applied to the local region of pain in the ankle. TENS was continued at home as before.

On the third treatment the patient reported $0 / 10$ on the VAS pain score, she presented with full movement of dorsiflexion and eversion, normal temperature in the foot and was fully weight bearing and the crutches had been discarded. There was still some tenderness in the lateral collateral ligament region. The treatment that followed was then local ultrasound to the affected region, rehabilitation in the pool and on land to improve balance and co-ordination. The patient was advised to continue with the orthotics and no further treatment was required.

\section{Discussion}

This patient was also referred as CRPS (previously RSD) "ankle". A literature review has revealed a study by Stilz et al (1977) that indicated successful treatment with TENS of RSD in children. The author has also found a very high percentage of success with TENS in CRPS in children therefore it was mandatory to treat this patient with TENS at home for at least 3 days for 8 hours per day. With this regimen Berger (1999) has been able to detect the com- plete and partial TENS responders in a pilot study and in many patients in the clinical situation in the minimum time of 2 to 3 days.

The previous history over the past two years of injury to the ankle region and then long periods without support to the foot (orthotics) during the 2002 holidays, predisposed the development of CRPS in this patient. The pain became generalised and the focal region was then disguised by unlocalised aching pain. The conventional physiotherapy treatment increased pain and eventually the patient was unable to weight bear. The distressing symptoms that then ensued - burning pain, night pain, inability to weight bear, cold foot and hyperaesthesia created a change in the child's personality and she became withdrawn, miserable and depressed. At the first treatment laser acupuncture was selected on the relevant points in order not to create fear of the treatment or any further discomfort and to engender confidence in the treatment. Laser treatment was applied to the local region (nonpainful) and a direct current (APS) applied to the whole leg to improve general circulation to the ankle. It is important not to increase pain during a treatment for this condition because any increase in pain or discomfort will irritate nerve endings and increase the pain and cause the condition to deteriorate. It was important to explain to the patient the type and reasons for the various applications of treatment and also the expected outcome of success that other children with this type of pain syndrome had obtained. This would hopefully also engender the placebo response which relies to a certain extent on the belief system and the confidence of the patient in the treatment. After the first treatment there was greater than 50\% improvement in the pain with a change in the child's personality according to the mother's description of "having her child back"! The pain had also localised to the lateral collateral ankle ligament and was then treated locally. At the third consultation the child was pain free and had discarded her crutches spontaneously. The patient then required balance, co-ordination and strength training in order to prevent recurrences. 


\section{CONCLUSION}

Patients that are detected early with any of the previously mentioned signs, symptoms and history should be viewed with suspicion of developing into CRPS contenders. These patients should initially receive pain blocking mechanisms and then be rehabilitated carefully back to normal, to prevent exacerbation. If sensitivity presents in a condition the normal physiotherapeutic assessment and treatment should be avoided and active assessment, observation of the area and distal treatment will enable the physiotherapist to analyse the problem, measure progress, develop the confidence of the patient in the treatment and ultimately treat the condition more effectively. When the condition becomes treatable all attempts must be made to find the cause and relieve and remove all inflammatory or indurated regions to prevent recurrence.

\section{REFERENCES:}

Babbedge R, Dray A, Urban L 1995 Complex regional pain syndromes (CRPS): mechanisms and therapy from experimental models. Pain Reviews 2: 298 - 309

Bennett G J 1994 Chapter 10: Neuropathic Pain. In: Textbook of Pain. Wall P D, Melzak R (editors) third edition. Churchill Livingstone, Edinburgh: 201

Berger P 1999 The role of the physiotherapist in the treatment of complex peripheral pain syndromes. Pain Reviews 6: 211-232

Birklein F, Kunzel W, Sieweke N 2001 Despite clinical similarities there are significant differences between acute limb trauma and CRPS 1 Pain 93(2): 165 - 171

Bruehl S, Harden R N, Galer BS, Saltz S, Backonja M, Stanton-Hicks M 2002 Complex regional pain syndrome: Are there distinct subtypes and sequential stages of the syndrome? Pain 95: 119 - 124

Del Carmen R S 1991 Reflex sympathetic dystrophy. Internal Anaesthesiology Clin. 29: 79 - 91

Devor M 1994 Chapter 4: The pathophysiology of damaged peripheral nerves. In: Textbook of Pain. Wall P D, Melzack R (editors) third edition. Churchill Livingstone, Edinburgh: $80-82$

Glynn C 1995 Complex regional pain syndrome type 1, reflex sympathetic dystrophy, and complex regional pain syndrome type II, causalgia. Pain Reviews 2: 292 - 297

Kidd B L, Morris V H, Urban L 1996 Pathophysiology of joint pain. Annals of Rheumatolgy Disease 55: 276 - 283

Kirk E J 1974 Impulses in dorsal spinal nerve rootlets in cats and rabbits arising from dorsal root ganglia isolated from the periphery. Journal of Comparative Neurology 155: 165 - 176

Mersky H, Bogduk N 1994 Classification of chronic pain. Seattle, IASP Press 2(41-42): 210 - 212

Mitchell S W, Moorhouse G R, Keen W W 1864 Gunshot injuries and other injuries to nerves. Philadelphia, PA: Lippincott Cited in: Bonica JJ. The management of pain, causalgia and other reflex sympathetic dystrophies 1990 volume one, second edition. Philadelphia, PA: Lee and Febiger: p220

Rang H P, Bevan S 1994 Nociceptive peripheral neurons: cellular properties. In: Textbook of Pain. Wall P D, Melzack M (editors), third edition. Churchill Livingston, Edinburgh: 57 - 58
Roberts W J 1986 An hypothesis on the physiological basis of causalgia and related pains. Pain 24: 297 - 311

Scadding J W 1999 Complex regional pain syndrome. In: Textbook of Pain. Wall P D, Melzack R (editors), fourth edition. Churchill Livingston, Edinburgh: 835 - 849

Shipton E A 1993 Pain acute and chronic. Johannesburg: Witwatersrand University Press: 163

Stanton Hicks M 1999 Official Joint Satellite meeting in Budapest of the SIG: Pain and the Sympathetic Nervous System and the Autonomic Section of the German Neurological Society.

\section{Stanton Hicks M 2001 World Institute of Pain Congress. Istanbul}

Stilz R J, Carron H, Sanders DB 1977 Reflex sympathetic dystrophy in a six year old: successful treatment by transcutaneous nerve stimulation. Anaesthetics and Analgesia 56: 438 - 441

Torebjork E, Wahren L, Wallin G, Hallin R, Kolzenburg M 1995 Noradrenaline evoked pain in neuralgia. Pain 63: 11 - 20

Travell J G, Simons D G 1983 Myofascial pain and dysfunction: the trigger point manual volume one. Willliams and Wilkins, Baltimore M D: $11-18$

Wasner G, Baron R 2001 CRPS - a disorder with decreased sympathetic reflexes. In: Special Interest Group Newsletter of the IASP: Pain and the Sympathetic Nervous System

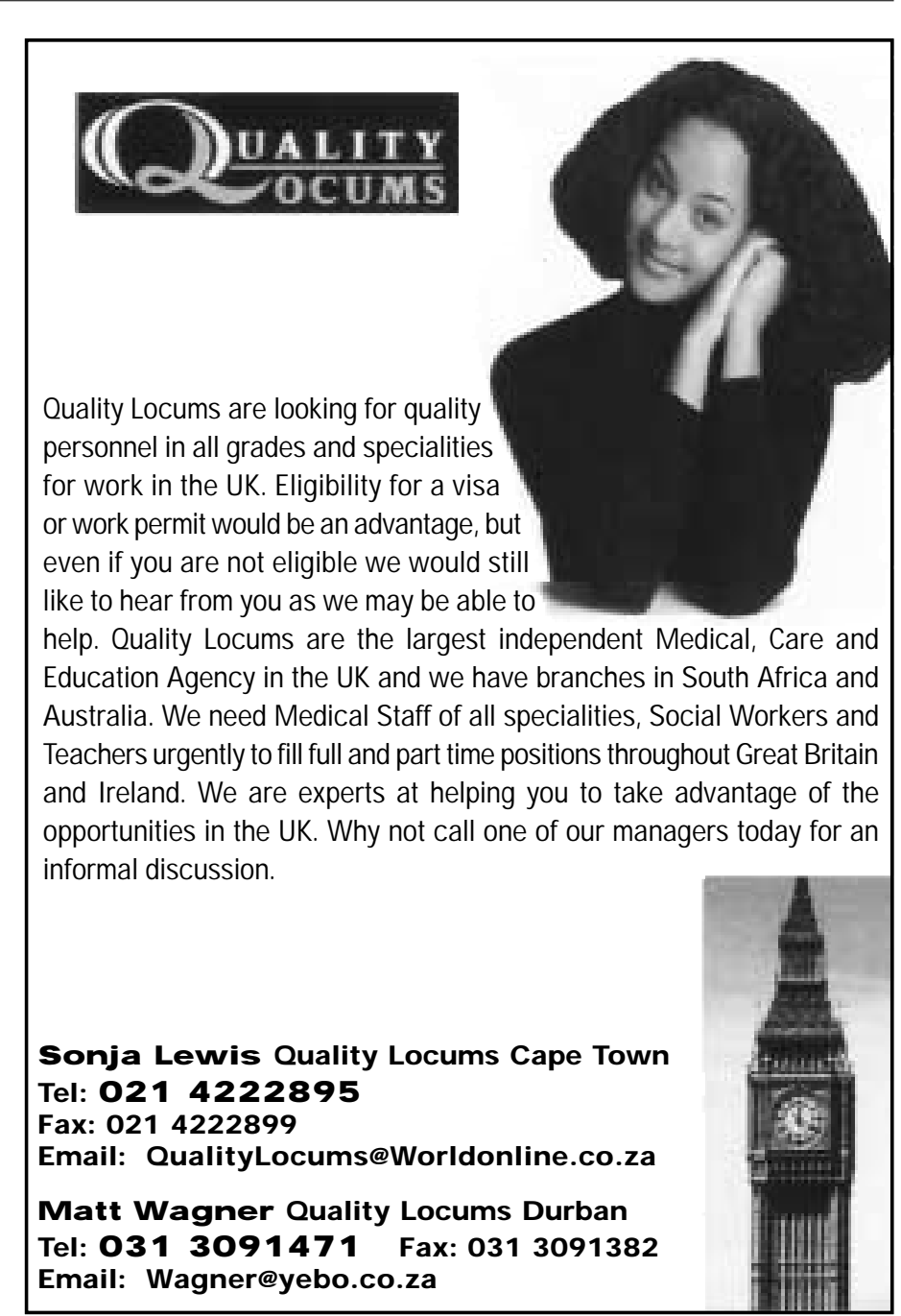

